\title{
Incidência e fatores de risco de lesões osteomioarticulares em corredores: um estudo de coorte prospectivo
}

CDD. 20.ed. 796.07

796.426

\author{
Paula PILEGGI* \\ Bruno GUALANO** \\ Maisa SOUZA* \\ Valéria de Falco CAPARBO* \\ Rosa Maria Rodrigues PEREIRA* \\ Ana Lucia de Sá PINTO* \\ Fernanda Rodrigues LIMA*
}

\section{Resumo}

A prática de corrida de média e longa distância tem crescido em todo o mundo. Apesar de todos os efeitos benéficos da prática de corrida, tem-se observado uma elevada incidência de lesões, sobretudo em membros inferiores. 0 mecanismo de lesão relacionada à corrida obedece a um padrão comum a todas as lesões nos diferentes esportes e decorre da sobreposição de dois ou mais fatores. Os objetivos desse estudo foram: 1) relatar prospectivamente a incidência de lesões osteomioarticulares em corredores amadores durante 12 meses de seguimento; e 2) detectar os principais fatores extrinsecos e intrínsecos para as lesões encontradas. Dezoito corredores (13 homens e cinco mulheres) amadores foram selecionados para participarem do estudo. Eles foram submetidos a uma avaliação clínica com exame físico completo e do aparelho locomotor, avaliação nutricional, exames laboratoriais, teste ergométrico, avaliação da densidade mineral óssea e composição corporal e radiografia dos pés no período basal e após um ano de seguimento. Aqueles que apresentaram alguma lesão foram comparados com seus pares que não lesionados, considerando-se as diversas variáveis coletadas. Metade da amostra (50\%) apresentou alguma lesão osteomuscular em membros inferiores no periodo do estudo. Os fatores de risco significantemente associados foram graus de extensão de joelho e flexão plantar diminuídos, frequência cardíaca de repouso menor e velocidade de treino maior. A alta frequência de lesões osteomioartculates nestes corredores de longa distância esteve associada a fatores intrínsecos e extrínsecos. A avaliação clínica deve ser focada nesses parâmetros com intuito de prevenir lesões em corredores.

Unitermos: Corrida; Lesão; Condicionamento físico; Alongamento; Osteoartrite.

\section{Introdução}

A prática de corrida de média e longa distância tem crescido em todo o mundo. De fato, diversas evidências indicam que o treinamento regular de corrida está associado à melhora na sensibilidade à insulina, reduções na quantidade de gordura corporal e concentrações de triglicérides, LDL e colesterol total, aumentos de massa magra e óssea, potência aeróbia e capacidade antioxidante, redução de pressão arterial pós-exercício e, como consequência dos fatores citados, melhora na qualidade de vida (para uma abrangente revisão, ver Pedersen e Saltin,
2006). Esses conhecimentos revelam que a prática dessa modalidade é de suma importância na prevenção primária, secundária e terciária de doenças crônicas, tais como: diabetes do tipo II, insuficiência cardíaca congênita, hipertensão arterial, obesidade, osteopenia, osteoporose, osteoartrite, entre outras (Pedersen \& Saltin, 2006).

Nesse sentido, a American College of Sports Medicine (ACSM) e American Heart Association (AHA) preconizam a prática regular de atividades físicas cíclicas, de longa duração, moderada 
intensidade e com o envolvimento de grandes grupamentos musculares, cujas características se enquadram perfeitamente à corrida.

Apesar de todos os efeitos benéficos da prática de corrida, tem-se observado uma elevada incidência de lesões no aparelho locomotor, sobretudo em membros inferiores (joelhos, quadril, tornozelos e pés). Uma recente meta-análise revelou que a incidência de lesôes em praticantes de corrida varia de 19,4\% a 79,3\% (Van Gent, Siem, Van Middelkoop, Van Os, Bierma-Zeinstra \& Koes, 2007). O mecanismo de lesão relacionada à corrida obedece a um padrão comum a todas as lesões nos diferentes esportes e decorre da sobreposição de dois ou mais fatores. De acordo com WEN (2007), esses fatores podem ser divididos em extrínsecos ou intrínsecos.

Os fatores extrínsecos são aqueles direta ou indiretamente ligados à preparação ou prática da corrida per se e envolvem 1) erros de planejamento e execução do treinamento (intensidade, frequência, duração, descanso, periodização, prática de aquecimento e alongamento); 2) tipo de superfície de treino (areia, asfalto, grama, concreto); 3) tipo de percurso (declive, tortuoso, plano, irregular); 4) tipo de calçado; 5) alimentação (consumo energético total, macro e micronutrientes, álcool ou drogas); 6) prática concomitante de outras modalidades esportivas. Já os fatores intrínsecos que supostamente predispõe às lesões são aqueles inerentes ao organismo e incluem 1) anormalidades biomecânicas e anatômicas (pés, tornozelo, calcâneo, tíbia, joelhos, assimetrias de comprimento dos membros inferiores); 2) flexibilidade; 3) histórico de lesōes; 4) características antropométricas (peso, altura, IMC); 5) densidade óssea e composição corpórea; 6) condicionamento cardiovascular (GELLMAN \& BURNS 1996; WEN, 2007). Alguns trabalhos investigaram os possíveis fatores de risco para lesões em atletas de alto nível ou militares submetidos a intensos programas de treinamento (BALlas, James, TYTKO \& Cookson, 1997; Giladi, Milgrom, Simkin \& Danon, 1991; Kaufman, Brodine, ShafFer, Johnson \& Cullison, 1999; Marti, Cader, Minder \& Abelin, 1988; O’Toole, 1992). No entanto, esses trabalhos focaram-se em: 1) único ou poucos fatores de risco para as lesōes ou 2) diversos fatores de risco para um único tipo de lesão, impossibilitando uma explicação abrangente sobre o tema. Além disso, atletas amadores são atualmente os que mais praticam a corrida. Comparados aos atletas de elite, corredores amadores geralmente cumprem menores volumes de treinamento e competição, porém podem estar sujeitos, por exemplo, a preparações físicas e nutricionais inadequadas. Especula-se, portanto, que os fatores capazes de explicar a incidência de lesōes em ambas as populações sejam distintos. Dessa forma, a investigação dos fatores predisponentes às lesões em atletas recreacionais é de grande valia.

Tendo em vista que a prevenção primária de lesões requer previamente a identificação dos fatores de risco aos quais uma população é exposta,os objetivos desse estudo foram 1) relatar prospectivamente a incidência de lesões osteomioarticulares em corredores amadores durante 12 meses de seguimento, e 2) detectar os principais fatores extrínsecos e intrínsecos para as lesões encontradas.

\section{Material e métodos}

\section{Amostra}

O presente estudo foi aprovado pelo Comitê de Ética do Hospital das Clínicas da Universidade de São Paulo (CAPPesq).

Após lerem e assinarem o termo de consentimento livre e esclarecido, 18 corredores (13 homens e cinco mulheres) amadores foram selecionados para participarem do estudo. Todos eles eram engajados em programas de corrida há pelo menos três meses, tinham como meta ao menos $50 \mathrm{~km}$ de distância percorrida por semana e treinavam, no mínimo, cinco vezes semanalmente. Os critérios de exclusão foram os seguintes: histórico prévio de cirurgia de membros inferiores; lesão osteomioarticular presente de membros inferiores; uso de esteróides anabolizantes, hormônio de crescimento ou outras substâncias consideradas ilícitas pelo Comitê Olímpico Brasileiro; histórico de doença ou medicações que influenciam a densidade óssea; participação em outra modalidade esportiva.

\section{Protocolo experimental}

Foi conduzido estudo de coorte prospectivo. Os participantes do estudo (13 homens e cinco mulheres) foram acompanhados durante 12 meses e avaliados para os parâmetros citados abaixo no 
período basal e no final de 12 meses. Os treinos ocorreram em grupo no Parque do Ibirapuera.

As lesões foram sempre comunicadas ao pesquisador por telefone ou pessoalmente no ambulatório de Medicina Esportiva da Disciplina de Reumatologia do Hospital das Clínicas, onde foram atestadas por avaliação médica sempre pelo mesmo pesquisador.

Ao término do seguimento, a amostra foi reagrupada de acordo com a presença ou não de lesões, com o intuito de comparar os grupos e, assim, determinar a influência de cada possível fator de risco na incidência das lesões.

\section{Avaliações clínicas}

Os atletas foram submetidos a um questionário detalhado que incluiu dados de fatores de risco referentes a seus hábitos diários e à prática de corrida, exame físico geral e osteomioarticular, conforme detalhado nos próximos subtópicos. Foram excluídos todos os atletas que apresentaram qualquer queixa osteomioarticular no início do projeto.

As lesōes foram classificadas de acordo com a interferência no treinamento (MARTI et al., 1988) em grau I (sem interrupção); grau II (redução do volume de treinamento); grau III (interrupção com duração de pelo menos duas semanas) e quanto à sintomatologia (GELLMAN \& BuRNs, 1996): agudas (persistência dos sintomas até duas semanas); subagudas (sintomas durando de duas a seis semanas) e crônicas (sintomas por mais de seis semanas).

\section{Dados gerais}

Foram colhidos dados de idade, doenças existentes, cirurgias e lesões prévias osteomioarticulares, consumo de bebidas alcoólicas, tabagismo, uso de suplementos alimentares, vitaminas, anabolizantes e outros hormônios. No caso das atletas femininas, foram também coletados dados sobre idade da menarca, alterações menstruais e uso de pílulas anticoncepcionais.

\section{Fatores de risco relacionados à corrida}

Foram colhidas informações referentes ao tempo de treino, quilometragem semanal, horas de treino semanal, velocidade, sequência de treinamento (aquecimento, alongamento, exercícios educativos, corrida propriamente dita e alongamento final), tipo de percurso e terreno de treino, prática de atividades alternativas (outras atividades físicas aeróbias e anaeróbias), informações sobre o calçado esportivo (tipo, tempo de uso, número de pares, critérios de compra, uso concomitante de órtese), tempo de prática de corrida e a experiência prévia em outras modalidades esportivas.

\section{Exame físico geral e esteomioarticular}

A estatura foi determinada usando um estadiômetro marca Sanny com resolução em milímetros. O peso foi obtido por meio de uma balança digital da marca Filizola. A partir dessas medidas, o índice de massa corporal (IMC) foi calculado usando a fórmula do peso dividido pela altura ao quadrado.

A amplitude articular foi determinada por goniometria tendo como referência os valores de normalidade abaixo especificados para cada articulação. O goniômetro usado nesse estudo foi do tipo "standart" ou de bolso com dois braços que giram em torno de um eixo central, estando em um dos braços marcados os graus de medição. Ao mover os braços do goniômetro, mensura-se a mobilidade articular. Foram medidos os ângulos do tornozelo em dorsiflexão e flexão plantar $(13 \pm 4,4$ e $56 \pm 6,1$ graus, respectivamente), dos joelhos em flexão e extensão (141 \pm 5,3 e 0-2 \pm 3 graus, respectivamente) e dos quadris em rotação externa e interna (32 $\pm 932 \pm 8$ graus, respectivamente).

$\mathrm{O}$ mesmo avaliador executou o exame físico inicial e final. A inspeção estática inicia-se com a observação do paciente em pé, em posição anatômica, de frente e perfil. Observou-se o alinhamento dos membros inferiores, joelhos e tornozelos, no mesmo plano (varo ou valgo), alinhamento patelar e ângulo "Q". $\mathrm{O}$ ângulo "Q" foi determinado por meio de uma linha imaginária conectando o centro da patela à espinha ilíaca Antero-superior, e uma outra linha em direção da inserção do tendão patelar na tuberosidade anterior da tíbia). A variação normal desse ângulo é de 13 a $18^{\circ}$ (Hoppenfeld \& Hutton, 1976). O joelho foi consiederado valgo quando o ângulo foi superior a $18^{\circ}$ e varo quando o ângulo foi inferior a $13^{\circ}$.

Para avaliação do encurtamento dos músculos isquiotibiais, o paciente se manteve em decúbito dorsal, em uma mesa de exame, com o quadril posicionado em 90 graus de flexão e com o joelho em flexão máxima. A partir desse ponto, o examinador executa a extensão passiva do joelho. Os músculos isquiotibiais foram considerados encurtados quando a extensão passiva foi inferior a $15^{\circ}$. Para avaliação do encurtamento do músculo quadríceps, o paciente se manteve em decúbito ventral, em uma mesa de exame, com o quadril posicionado em 0 grau, o joelho posicionado em extensão máxima e a face anterior da coxa em contato com a mesa de 
exame. A partir desse ponto, o examinador executa a flexão passiva do joelho. O músculo quadríceps foi considerado encurtado quando o calcanhar não alcançou a região glútea na flexão passiva da perna (Hoppenfeld \& HutTon, 1976).

\section{Exames laboratoriais}

Os exames laboratoriais foram colhidos no início e ao final dos 12 meses de seguimento. Foram obtidos hemograma completo e concentrações sanguíneas de ferro, ferritina e cortisol.

\section{Teste ergométrico}

Os atletas foram submetidos à ergometria apenas antes do seguimento. A esteira ergométrica utilizada foi a Embramed $囚$. O programa utilizado foi o ERGO PC13 (Micromed®) e o protocolo de aptidão cardiorrespiratória adotado foi Ellestad. Os parâmetros avaliados foram: frequência cardíaca (FC) máxima e estimativa do consumo máximo de oxigênio ( $\left.{ }^{\mathrm{N}} \mathrm{O}_{2} \max \right)$.

\section{Densitometria por Raios X (DEXA)}

A composição corporal foi avaliada apenas no período basal por meio da análise densitométrica de corpo total, utilizando-se densitômetro QDR2000. Os compartimentos corporais avaliados foram conteúdo mineral ósseo total (CMO), massa magra e massa gorda. A porcentagem de gordura total foi obtida através da relação entre a massa gorda e o peso total estabelecido pelo densitômetro.

\section{Avaliação radiológica}

As medidas de ângulos dos pés foram feitas através de raio x. O ângulo formado por duas linhas que vão, respectivamente, da base do sesamóide do primeiro dedo ao ponto mais inferior do tálus e do ponto mais inferior do tálus ao ponto mais inferior do calcâneo.

Os pés cavos ou planos foram avaliados pelo raio $\mathrm{x}$ dos pés em perfil, ortostático e com carga. Foram considerados normais ângulos de 120 a 125 graus.

\section{Avaliação nutricional}

Foram empregados recordatórios de 24 horas com intuito de obter informações acerca do consumo alimentar dos voluntários. Brevemente, cada atleta foi instruído a relatar sistematicamente ao entrevistador o tipo e a quantidade de alimentos consumidos no dia anterior a entrevista.

\section{Análises estatísticas}

As variáveis qualitativas foram expressas em frequência absoluta (n) e relativa (\%) e as quantitativas por média e desvio padrão (dp). As variáveis qualitativas (nominais) foram comparadas pelo teste do Qui-quadrado $\left(\mathrm{x}^{2}\right)$ ou Teste Exato de Fisher.

A distribuição normal das variáveis quantitativas foi avaliada pelo teste de Kolmogorov-Smirnov. As variáveis não paramétricas foram comparadas pelo teste de Mann-Whitney para medidas independentes. Já as variáveis paramétricas, avaliadas somente no período basal, foram analisadas por meio do teste $\mathrm{T}$ de Student para medidas independentes.

A Análise de Variância (ANOVA) para medidas repetidas foi utilizada para comparações basais e ao longo do tempo para variáveis paramétricas. Para variáveis não paramétricas, empregou-se o teste de Wilcoxon.

$\mathrm{O}$ nível de significância adotado para se rejeitar a hipótese nula foi de $\mathrm{p} \leq 0,05$. Valores de $\mathrm{p} \leq 0,1$ foram considerados tendência à significância. As análises foram conduzidas com auxílio do programa SPSS.

\section{Resultados}

A média de peso e estatura da amostra estudada foi de $61,8 \pm 10,2 \mathrm{~kg}$ e $170,5 \pm 9,5 \mathrm{~cm}$, respectivamente.

Após 12 meses de seguimento, nove corredores (50\%) apresentaram 22 lesões osteomioarticulares. Foram elas: fratura de estresse em crista ilíaca esquerda, lesão da musculatura adutora esquerda, síndrome da banda íliotibial bilateralmente, tendinopatia patelar bilateralmente, bursite infrapatelar esquerda, periostite tibial bilateralmente, síndrome do estresse tibial à esquerda, entesopatia tibiais, fraturas de estresse tibial bilateralmente, lesão da musculatura da panturrilha esquerda, tendinite aquileana direita, bursite retrocalcânea direita, fasciíte plantar esquerda (TABELA 1). 
TABELA 1 - Eventos observados após 12 meses de seguimento.

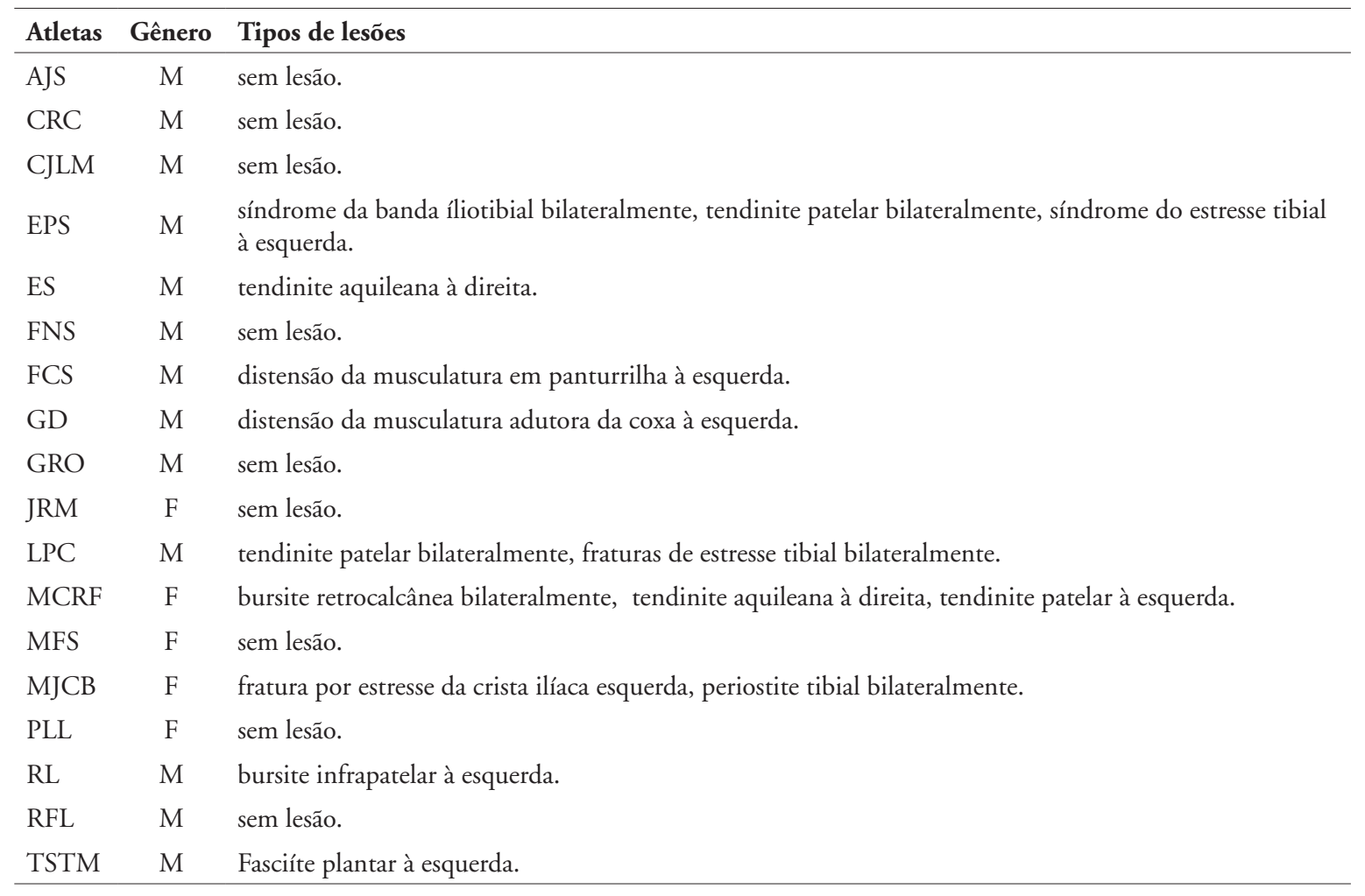

Dentre os nove corredores lesionados, quatro deles apresentaram três ou mais lesões. Nenhum apresentou lesōes de grau I, seis $(66,7 \%)$ apresentaram lesōes de grau II e três $(33,3 \%)$ tiveram lesōes de grau III. Em relação à época das lesōes, quatro $(44,4 \%)$ tiveram lesōes durante o treino, um $(11,1 \%)$ durante o aquecimento, um $(11,1 \%)$ após o treino, um $(11,1 \%)$ após o aquecimento e durante o treino, um $(11,1 \%)$ durante treino e prova e um $(11,1 \%)$ durante o treino e após competir. A maior ocorrência de lesóes aconteceu durante o treino, em sete $(77,8 \%)$ dos atletas. No que se refere à sintomatologia, dois $(22,3 \%)$ apresentaram lesões agudas, quatro $(44,4 \%)$ com lesōes subagudas e três (33,3\%) lesōes crônicas.

A análise comparativa dos dados demográficos entre os grupos lesão e sem lesão não mostrou diferença significativa com relação à média de idade, sexo, raça e renda mensal. Da mesma forma, não houve diferença significativa com relação aos parâmetros antropométricos (peso, estatura e IMC).

Não houve diferença significativa na análise comparativa entre os grupos com relação ao alinhamento dos joelhos. Também não foi observada diferença significativa de efeito de grupo e de amostra total na avaliação goniométrica com relação aos seguintes parâmetros: joelho direito em extensão, quadril esquerdo e direito em rotação externa, quadril esquerdo em rotação interna, dorsiflexão plantar esquerda e direita, flexão plantar direita.
Não houve diferença entre os grupos com e sem lesão encurtamento muscular. No entanto, encontramos diferença estatisticamente significante com relação ao joelho esquerdo em extensão (menor amplitude no grupo lesionado) e em flexão (menor amplitude na avaliação final da amostra total), quadril direito em rotação interna (menor amplitude na avaliação final da amostra total), flexão plantar esquerda (maior amplitude no grupo lesionado) conforme demonstrado na TABELA 2.

Não houve diferenças significantes entre os grupos para os demais parâmetros intrínsecos avaliados, tais como: $\mathrm{da}$ dos laboratoriais (hemoblobina, ferritina, cortisol e ferro), densidade mineral óssea e composição corporal, potência aeróbia ( $\mathrm{W}_{2}$ max), dados radiológicos (pés normais, cavos ou planos), ingestão total e de macronutrientes (proteína, carboidrato e lipídio) (TABELA 2).

A frequência cardíaca de repouso foi menor no grupo de corredores que apresentaram lesão quando comparados aos seus pares não lesionados. As demais variáveis referentes à corrida (tempo de treino e distância percorrida semanalmente, média de velocidade, tipo de percurso e superfície de treino, número e tempo de uso de pares de tênis, uso de órteses, número de competiçôes, tempo de alongamento, lesões prévias, prática atual ou anterior de outras atividades físicas, tempo de prática da corrida) não foram diferentes entre os grupos (TABELA 3). 
${ }^{* *} p<0,05$ - efeito principal de tempo (Pré vs. Pós);

${ }^{*} p<0,05$ - efeito de interação (grupo com lesão vs. grupo sem lesão);

\# 0,1 > p >0,05 - tendência à interação (grupo com lesão vs. grupo sem lesão). esq. = esquerdo; dir. = direito; MMII = membros in feriores.
TABELA 2 - Parâmetros clínicos, radiográficos, nutricionais, de composição corporal e de capacidade física relacionados à lesão.

\begin{tabular}{lcccc}
\hline & \multicolumn{2}{c}{ Grupo sem lesóes } & \multicolumn{2}{c}{ Grupo com lesóes } \\
\cline { 2 - 5 } & Pré & Pós & Pré & Pós \\
\hline IMC (kg/m $)$ & $17,4 \pm 1,7$ & $18,6 \pm 1,9$ & $18,2 \pm 2,9$ & $18,3 \pm 3$ \\
Alinhamento de joelhos & $5(55,6)$ & & $2(22,2)$ & \\
Raio X, n (\%) & & & & \\
Pés planos & 0 & & $2(22,2)$ & \\
Pés cavos & $3(33,3)$ & & $6(66,7)$ & \\
Goniometria (graus), m \pm dp & & & & \\
Joelho esq. em extensão & $5,7 \pm 2,3^{*}$ & $5,3 \pm 2,9$ & $3,5 \pm 1,4$ & $3,6 \pm 2,2$ \\
Joelho dir. em extensão & $5,2 \pm 1,8$ & $6,2 \pm 2,5$ & $6,1 \pm 1,7$ & $4,7 \pm 1$ \\
Joelho esq. em flexão & $133,7 \pm 5,8^{* *}$ & $129,5 \pm 2,3$ & $133,1 \pm 3,5^{* *}$ & $131 \pm 4,4$ \\
Joelho dir. em flexão & $133,1 \pm 7,2^{* *}$ & $129,1 \pm 3,2$ & $132 \pm 3,6^{* *}$ & $129,4 \pm 4,8$ \\
Quadril esq. em rotação externa & $31,5 \pm 2,8$ & $29,1 \pm 3,7$ & $29,8 \pm 6,2$ & $28,3 \pm 4,8$ \\
Quadril dir. em rotação externa & $32,7 \pm 3,8$ & $28,7 \pm 4,9$ & $28,5 \pm 4,6$ & $29,4 \pm 4$ \\
Quadril esq. em rotação interna & $31,7 \pm 5,6$ & $28,4 \pm 4,1$ & $31,5 \pm 3,6$ & $31,5 \pm 4,4$ \\
Quadril dir. em rotação interna & $33,2 \pm 3,6^{* *}$ & $28,8 \pm 4,1$ & $30,4 \pm 5,1 * *$ & $29,1 \pm 5$ \\
Dorsiflexão plantar esq. & $18,4 \pm 2,3$ & $17,1 \pm 3$ & $17,6 \pm 4,4$ & $17,2 \pm 2,9$ \\
Dorsiflexão plantar dir. & $16,4 \pm 3,2$ & $17,4 \pm 3,6$ & $16,6 \pm 3,8$ & $17,1 \pm 3,3$ \\
Flexão plantar esq. & $47,4 \pm 4,9^{*}$ & $50 \pm 4,8$ & $54,3 \pm 6,4$ & $50,3 \pm 7,6$ \\
Flexão plantar dir. & $47,8 \pm 5,6$ & $48,3 \pm 5,1$ & $50,4 \pm 7,3$ & $51,7 \pm 5,2$
\end{tabular}

Encurtamento muscular, $\mathbf{n}(\%)$

Quadríceps

$7(77,8)$

$8(88,9)$

$5(55,6)$

$5(55,6)$

0

$3(33,3)$

$0,06 \pm 0,17$

$0,67 \pm 0,43$

Flexibilidade MMII (graus), n (\%)

Esq.

$81,8 \pm 50,9^{*}$

$66,6 \pm 8,2$

Dir.

$81,8 \pm 50,9^{*}$

$66,8 \pm 8,9$

\section{Parâmetros laboratorais}

Hemoglobina (g/dl)

Ferro $(\mu \mathrm{g} / \mathrm{dL})$

Ferritina $(\mathrm{ng} / \mathrm{mL})$

Cortisol $(\mu \mathrm{g} / \mathrm{dL})$

$\begin{array}{cccc}13,9 \pm 1,8 & 13,9 \pm 1 & 14,3 \pm 1,1 & 13,9 \pm 1,4 \\ 111,6 \pm 34,7 & 101,5 \pm 30,1 & 93,5 \pm 26,1 & 99,8 \pm 44,8 \\ 46,7 \pm 27,6 & 40,8 \pm 28,3 & 88,68 \pm 82,3 & 80,4 \pm 86,5 \\ 11,7 \pm 3,3 & 11,3 \pm 3,7 & 12,9 \pm 7,8 & 13,3 \pm 2,5\end{array}$

Capacidade aeróbia

نं $\mathrm{O}_{2} \max (\mathrm{ml} / \mathrm{kg} / \mathrm{min})$

$69 \pm 13,6 \#$

$183,1 \pm 9,3$

$77,5 \pm 10,4$

FCmax (bpm/min)

$63,3 \pm 7,4^{*}$

$183,8 \pm 7,3$

FC de repouso (bpm/min)

\section{Composição corporal}

Conteúdo mineral ósseo

$2,53 \pm 0,6$

$2,39 \pm 0,4$

Densidade mineral óssea

$1,16 \pm 0,13$

$1,11 \pm 0,09$

Gordura corporal (\%)

$15,2 \pm 8$

$14,9 \pm 10,4$

$80,5 \pm 7,5$
$81,1 \pm 9,9$ 
TABELA 2 - Parâmetros clínicos, radiográficos, nutricionais, de composição corporal e de capacidade física relacionados à lesão (continuação).

\begin{tabular}{lcccc}
\hline & \multicolumn{2}{c}{ Grupo sem lesóes } & \multicolumn{2}{c}{ Grupo com lesóes } \\
\cline { 2 - 5 } & \multicolumn{1}{c}{ Pré } & Pós & Pré & Pós \\
\hline Consumo alimentar & & & & \\
Carboidratos (g) & $326 \pm 38$ & $264 \pm 38$ & $400 \pm 258$ & $265 \pm 67$ \\
Carboidratos (\%) & $53,9 \pm 9,3$ & $54,3 \pm 9,9$ & $49,2 \pm 12,2$ & $52,5 \pm 8,5$ \\
Proteínas (g) & $118 \pm 67^{* *}$ & $94 \pm 66$ & $149 \pm 67^{* *}$ & $97 \pm 18$ \\
Proteínas (\%) & $18,29 \pm 6,12$ & $17,31 \pm 7,7$ & $20,90 \pm 8,2$ & $19,5 \pm 3$ \\
Lipídeos (g) & $84 \pm 32^{* *}$ & $64 \pm 27$ & $103 \pm 55^{* *}$ & $61 \pm 17$ \\
Lipídeos (\%) & $30,6 \pm 5,6$ & $28,3 \pm 7,9$ & $29,8 \pm 7,2$ & $27,8 \pm 7,6$ \\
Calorias totais (kcal) & $2427 \pm 1048^{* *}$ & $1946 \pm 460$ & $3101,7 \pm 1359^{* *}$ & $1991 \pm 298$ \\
\hline
\end{tabular}

${ }^{* *} p<0,05$ - efeito principal de tempo (Pré vs. Pós);

${ }^{*} p<0,05$ - efeito de interação (grupo com lesão vs. grupo sem lesão);

\# $0,1>p>0,05-$ tendência à interação (grupo com lesão vs. grupo sem lesão). esq. = esquerdo; dir. = direito; MMII = membros in feriores.

TABELA 3 - Fatores da prática de corrida relacionadas à lesão.

\section{Grupo sem lesões}

Dados relacionados à corrida

Distância percorrida $(\mathrm{km} / \mathrm{sem}), \mathrm{m} \pm \mathrm{dp}$

Tempo de treino ( $h / s e m), m \pm d p$

Velocidade $(\mathrm{min} / \mathrm{km}), \mathrm{m} \pm \mathrm{dp}$

Tipo de percurso, $\mathrm{n}(\%)$

Misto

1 ou 2 tipos

Tipo de terreno, n (\%)

Misto

1 ou 2 tipos

Número de pares de treino, $\mathrm{n}(\%)$

3 ou mais

1 ou 2

Duração do tênis, n (\%)

Mais de $800 \mathrm{~km}$

Uso de órteses, n (\%)

Tempo de alongamento, $\mathrm{n}(\%)$

10 a $15 \mathrm{~min}$

20 a $30 \mathrm{~min}$

Número de competições, $\mathrm{m} \pm \mathrm{dp}$

Práticas atividades alternativas, $\mathrm{n}(\%)$

Tempo de prática de corrida, n (\%)

Mais de 12 meses

Menos de 12 meses

Prática prévia (outros esportes), n (\%)
$3(33,3)$

$4(44,4)$

$5(55,6)$

$1(11,1)$

$6(66,7)$

$3(33,3)$

\section{Grupo com lesóes}

$$
\begin{gathered}
78,8 \pm 27,5 \\
8,7 \pm 1,9 \\
423 ” \pm 0,5
\end{gathered}
$$

$6(66,7)$

$2(22,2)$

$5(55,6)$

$8 \pm 4$

$2(22,2)$

$6(66,7)$

$3(33,3)$

$5(55,6)$
$2(22,2)$

$7(77,8)$

$8(88,9)$

$1(11,1)$

$2(22,2)$

$8(88,8)$

$1(11,1)$

$9 \pm 11$

$2(22,2)$

$9(100)$

0

$7(77,8)$ p > 0,1 para todos os dados. 


\section{Discussão}

O primeiro objetivo desse estudo foi determinar a incidência de lesões do aparelho locomotor numa coorte de indivíduos amadores durante seguimento de um ano. Os dados obtidos revelaram que a ocorrência de lesões foi alta (50\%), tal qual em uma coorte de atletas amadores holandeses, cuja incidência foi de 54,8\% (Van MiddelKoOp, Kolkman, Van Ochten, Bierma-Zeinstra \& Koes, 2008).

Esses resultados são muito preocupantes uma vez que as lesões do aparelho locomotor em corredores podem aumentar a incidência de osteoartrite (URQUHART, Wluka, Teichtahl \& CicutTini, 2007; Vingard, Alfredsson \& Malchau, 1998). Diante disso, uma importante diretriz sugere que todos os praticantes de esporte sejam alertados desse risco, que aumenta de acordo com a duração e intensidade da exposição (Vignon, Valat, Rossignol, Avouac, Rozenberg, Thoumie, Avouac, Nordin \& Hilliquin, 2006). Genericamente, essa recomendação seria de grande valia particularmente àqueles corredores que buscam melhoria na saúde, sem saber, no entanto, que podem estar sujeitos a complicações que, em última análise, prejudicariam sobremaneira à qualidade de vida.

Diante da alta incidência de eventos osteomioarticulares observados nesse e em outros estudos, esforços devem ser focados na prevenção dessas lesões. O primeiro passo nesse sentido, entretanto, deve ser a investigação dos eventuais fatores de risco predisponentes. Portanto, o segundo objetivo desse estudo foi examinar possíveis fatores extrínsecos e intrínsecos relacionados à ocorrência de lesões do aparelho locomotor na população estudada.

Em consonância com estudos prévios (CLEMENTS, Yates \& Curran, 1999; Wen, Puffer \& SchmalzRied, 1997), os dados demográficos, tais como idade e gênero não explicaram a incidência de lesões nesse estudo.

Os valores de IMC também não foram diferentes entre os grupos com e sem lesões, em linha com outros achados (GILADI et al., 1991). Em contrapartida, a literatura demonstrou um aumento da incidência de lesões em calcâneo paralelamente ao aumento do IMC (Wen, Puffer \& Schmalzried, 1997). Além disso, valores de IMC menores que 19,5 e maiores que 27 foram preditivos de lesões em corredores (MARTI et al., 1988). De fato, maiores valores de IMC estão associados à progressão radiográfica de osteoartrite em corredores de meia idade e idosos (Chakravarty, Hubert, Lingala, ZATARAIN \& Fries, 2008). A estreita variação de IMC apresentada pela amostra impossibilita a detecção de diferenças entre os grupos. Portanto, o erro do tipo II (aceitar a hipótese nula quando a mesma é falsa) não pode ser descartado para essa variável.

Conforme relatado em estudos prévios (CLEMENTS, YATES \& CURRAN, 1999; MARTT et al., 1988), joelho e tíbia foram as estruturas anatômicas mais afetadas pelas lesōes. Segundo alguns autores, joelhos valgos, varos e recurvados e tíbia vara são considerados fatores de risco para lesōes em corredores (COWAN, Jones, FrykMan, POLLY JunIOR, Harman, Rosenstein \& Rosenstein, 1997; Wen, 2007). No presente estudo, contudo, a presença de alteraçōes de alinhamento não foi diferente entre os grupos. A discrepância entre os achados em estudos prévios quanto ao alinhamento estático de membros inferiores como fator de risco para lesóes em corredores permanece, portanto, ainda não elucidada (WEN, 2007).

Há evidências indicando que o aumento do número de dias de treino e da distância percorrida por semana são fatores preditivos para lesões de membro inferior (SATTERTHWAITE, NORTON \& LARMER, 1999). No entanto, tais associações não foram possíveis de serem testadas no presente estudo, uma vez que não houve diferença significante para essas variáveis entre o período basal e após o seguimento.

Além disso, uma recente meta-análise demonstrou que as evidências para a influência de outras variáveis referentes à corrida (tempo de prática de corrida, tipos de terreno e percurso, número de pares e tempo de uso dos calçados esportivos, número de participação em eventos e prática de atividades alternativas) na incidência de lesões são muito limitadas (VAN MiddelKoop et al., 2008). Por outro lado, a mesma meta-análise encontrou uma importante evidência da presença prévia de lesōes como um fator de risco para o surgimento de novas lesões. O mesmo foi observado nos nossos resultados. As explicaçóes para esses achados contraditórios são especulativas, mas podem envolver diferenças nos tipos, graus e quantidade das lesôes prévias e recuperação total ou parcial das mesmas. Estudos futuros devem esclarecer o real papel desse fator de risco em lesões de corredores.

A distância semanal percorrida $(>65 \mathrm{~km})$ tem sido considerada um importante fator de risco predisponente à lesão (Walter, Hart, MCIntosh \& SUTtON, 1989). Nossos achados, entretanto, são opostos a estes previamente publicados. Embora os corredores tenham percorrido distâncias semanais médias superiores àquelas relatadas como fatores de risco, não houve diferenças entre os grupos. Contudo, nossos dados de maiores velocidades durante o treinamento 
(indicativo de intensidade de treino) e menores FC de repouso (indicativo de adaptação cardiovascular ao exercício físico) no grupo com lesão sugerem, coletivamente, que corredores submetidos a intensidades elevadas de treinamento estão, de fato, mais propícios às lesões. Em última análise, esses resultados estão de acordo com a possibilidade de que a incidência de osteoartrite de joelhos é proporcional à exposição e intensidade da prática esportiva (VIGNON et al., 2006).

Os graus de extensão de joelho e flexão plantar diminuídos foram fatores associados às lesões nesse estudo. Em contrapartida, o tempo de alongamento pareceu não influenciar na incidência de lesôes. Esses achados aparentemente contraditórios ilustram a discrepância existente na literatura. Em recente revisão, Woods, BISHOP e JONES (2007) sugerem que alguns protocolos de alongamento são efetivos em reduzir lesões e, por isso, recomendam que essa prática seja empregada 15 minutos antes de uma sessão de treino. Por outro lado, recentes revisões sistemáticas não encontraram efeitos benéficos de um programa de alongamento na prevenção de lesões (AALTONEN, KARJALAINEN, HeINOnen, ParkKari \& Kujala, 2007; Thacker, Gilchrist, STroup \& KimseY Junior, 2004). Mais estudos são necessários para desvendar o papel da flexibilidade na ocorrência de lesões, assim como de um programa de alongamento na prevenção das mesmas.

Outros fatores relacionados ao risco de lesões osteomioarticulares em corredores como consumo alimentar, alterações de postura e da marcha e parâmetros laboratoriais não explicam a incidência de lesões nesse estudo, bem como em outros trabalhos que avaliaram os mesmos fatores (VAN MidDELKOOP et al., 2008; WEN, 2007).

Uma limitação do nosso estudo foi o pequeno tamanho da amostra, o que não permitiu realizar análises multivariadas para atestar o peso de cada variável na ocorrência de lesão.

Em suma, o presente estudo demonstrou que a incidência de lesões em uma coorte de corredores brasileiros seguidos por um ano é de 50\%, compatível com a média encontrada mundialmente. Além disso, observou-se que os únicos fatores predisponentes às lesões foram flexibilidade, amplitude articular, FC de repouso e velocidade executada durante o treino. Dessa forma, são necessários novos estudos longitudinais com corredores brasileiros no sentido de se identificar precocemente os fatores de risco particulares à nossa população e com isso, minimizar o risco de lesôes.

\begin{abstract}
Incidence and risk factors of lower limb injury in runners: a prospective cohort study

The practice of middle- and long-distance running has become worldwide popular. Despite the number of benefits associated with this sport, increased incidence of lower limb injury has been observed. The injury mechanisms related to running are similar to those seen in different sports and can be a result of two or more factors. The aims of this study were: 1 ) to report prospectively the incidence of injuries in non professional runners after a 12-month follow-up; and 2) to determine the main intrinsic and extrinsic factors related to the observed injuries. Eighteen runners (13 males and five females) took part in this study. They were submitted to clinical examination, nutritional and biochemical assessments, $\mathrm{VO}_{2}$ max test, bone mineral density and body composition evaluation, and foot radiography at baseline and after one year. The subjects who had injury were compared to those non-injured taken into account the several variables assessed. Fifth percent of the sample presented at least one lower limb injury. The factors significantly associated with the injuries were reduced knee extension and plantar flexion range of motion, lower resting heart rate, and high training speed. The high incidence of injuries observed in this study was associated with intrinsic and extrinsic factors. The clinical assessment should focus on these parameters in order to prevent injuries.
\end{abstract}

UnITERMS: Running; Injury; Physical conditioning; Stretching; Osteoarthritis.

\title{
Referências
}

AALTONEN, S.; KARJALAINEN, H.; HEINONEN, A.; PARKKARI, J.; KUJALA, U.M. Prevention of sports injuries: systematic review of randomized controlled trials. Archives of Internal Medicine, Chicago, v.167, p.1585-92, 2007. 
BALLAS, M.T.; TYTKO, J.; COOKSON, D. Common overuse running injuries: diagnosis and management. American Family Physician, Kansas City, v.15, n.55, p.2473-84, 1997.

CLEMENTS, K.; YATES, B.; CURRAN, M. The prevalence of chronic knee injury in triathletes. British Journal of Sports Medicine, London, v.33, p.214-6, 1999.

CHAKRAVARTY, E.F.; HUBERT, H.B.; LINGALA, V.B.; ZATARAIN, E.; FRIES, J.F. Long distance running and knee osteoarthritis: a prospective study. American Journal of Preventive Medicine, New York, v.35, p.133-8, 2008.

COWAN, D.N.; JONES, B.H.; FRYKMAN, P.N.; POLLY JUNIOR, D.W.; HARMAN, E.A.; ROSENSTEIN, R.M.; ROSENSTEIN, M.T. Lower limb morphology and risk of overuse injury among male infantry trainees. Medicine and Science in Sports and Exercise, Madison, v.28, p.945-52, 1996.

GELLMAN, R.; BURNS, S. Walking aches and running pains: injuries of the foot and ankle. Orthopedics, Thorofare, v.23, p.263-79, 1996. GILADI, M.; MILGROM, C.; SIMKIN, A.; DANON, Y. Stress fractures: identifiable risk factors. American Journal of Sports Medicine, Columbus, v.19, p.647-52, 1991.

HOPPENFELD, S.; HUTTON, R. Physical examination of the spine \& extremities. In: HOPPENFELD, S.; HUTTON, R. (Eds.). Physical examination of the knee, ankle, foot and lumbar spine. New York: Prentice Hall, 1976. p.171-264. KAUFMAN, K.R; BRODINE, S.K.; SHAFFER, R.A.; JOHNSON, C.W.; CULLISON, T.R. The effect of foot structure and range of motion on musculoskeletal overuse injuries. American Journal of Sports Medicine, Columbus, v.27, p.585-93, 1999. MARTI, B.; CADER, J.P.; MINDER, C.E.; ABELIN, T. On the epidemiology of running injuries: the 1984 Bern Grand -Prix study. American Journal of Sports Medicine, Columbus, v.16, p.285-94,1988.

O’TOOLE, M.L. Prevention and treatment of injuries to runners. Medicineand Sciencein Sports and Exercise, Madison, v.24, p.S360-3, 1992. PEDERSEN, B.K.; SALTIN, B. Evidence for prescribing exercise as therapy in chronic disease. Scandinavian Journal of Medicine and Science in Sports, Stockholm, v.16, p.3-63, 2006.

SATTERTHWAITE, P.; NORTON, R.; LARMER, P. Risk factors for injuries and other health problems sustained in a marathon. British Journal of Sports Medicine, London, v.33, p.22-6, 1999.

THACKER S.B.; GILCHRIST, J.; STROUP, D.F.; KIMSEY JUNIOR, C.D. The impact of stretching on sports injury risk: a systematic review of the literature. Medicine and Science in Sports and Exercise, Madison, v.36, p.371-8, 2004. URQUHART, D.; WLUKA, A.; TEICHTAHL, A.; CICUTTINI, F. The effect of physical activity on the knee joint: is it good or bad? British Journal of Sports Medicine, London, v.41, p.546-7, 2007.

VAN GENT, R.N.; SIEM, D.; VAN MIDDELKOOP, M.; VAN OS, A.G.; BIERMA-ZEINSTRA, S.M.; KOES, B.W. Incidence and determinants of lower extremity running injuries in long distance runners: a systematic review. British Journal of Sports Medicine, London, v.41, p.469-80, 2007.

VAN MIDDELKOOP, M.; KOLKMAN, J.; VAN OCHTEN, J.; BIERMA-ZEINSTRA, S.M.; KOES, B. Prevalence and incidence of lower extremity injuries in male marathon runners. Scandinavian Journal of Medicine and Science in Sports, Stockholm, v.18, p.140-4, 2008.

VIGNON, E.; VALAT JP.; ROSSIGNOL, M.; AVOUAC, B.; ROZENBERG, S.; THOUMIE, P.; AVOUAC, J.; NORDIN, M.; HILLIQUIN, P. Osteoarthritis of the knee and hip and activity: a systematic international review and synthesis (OASIS). Joint Bone Spine, Paris, v.73, p.442-55, 2006.

VINGARD, E.; ALFREDSSON, L.; MALCHAU H. Osteoarthrosis of the hip in women and its relationship to physical load from sports activities. American Journal of Sports Medicine, Columbus, v.26, p.78-82, 1998.

WALTER, S.D.; HART, L.E.; McINTOSH, J.M.; SUTTON, J.R. The Ontario cohort study of running-related injuries. Archives of Internal Medicine, Chicago, v.149, n.11, p.2561-4, 1989.

WEN, D.Y. Risk factors for overuse injuries in runners. Current Sports Medicine Reports, Philadelphia, v.6, p.307-13, 2007. WEN, D.Y.; PUFFER, J.C.; SCHMALZRIED, T.P. Lower extremity alignment and risk of overuse injuries in runners. Medicine and Science in Sports and Exercise, Madison, v.29, p.1291-8, 1997.

WOODS, K.; BISHOP, P.; JONES, E. Warm-up and stretching in the prevention of muscular injury. Sports Medicine, Auckland, v.37, p.1089-99, 2007.

ENDEREÇO

Fernanda R. Lima

Faculdade de Medicina

Universidade de São Paulo

Av. Dr. Arnaldo, 455 - 3o. andar - Reumatologia ooooo-00o - São Paulo - SP - BRASIL e-mail: reumato@usp.br
Recebido para publicação: 01/09/2009

1a. Revisão: 29/03/2010

2a. Revisão: 10/05/2010

Aceito: 28/05/2010 